\title{
TF adaptive vs wave one gold - evaluation of shaping characteristics in the mesial canals of mandibular first molars-CBCT ex vivo study
}

\author{
Anil Dhingra ${ }^{1}$, Nayanika Singh Rathore, ${ }^{2, *}$ \\ ${ }^{\mathbf{1}}$ Professor \& HOD, ${ }^{2}$ Post Graduate Student, ${ }^{\mathbf{1}, 2}$ Dept. of Conservative Dentistry and Endodontics, Seema Dental College and \\ Hospital, Rishikesh, Uttarakhand, India \\ *Corresponding Author: \\ Email: drnayanikasingh@gmail.com
}

\begin{abstract}
AIM: This ex vivo study compared the shaping characterstics of Twisted File Adaptive and Wave One Gold file systems using Cone Beam Computed Tomography (CBCT).

Methodology: Sixty extracted human permanent mandibular first molars with mesial roots curvature angles ranging within 20-30 degrees were assigned into three groups ( $n=30$ each). Teeth were imaged ( 90 micron resolution) with CBCT imaging software CS9300 equipment set at $84 \mathrm{Kv}, 5 \mathrm{~mA}, 10.8$ second exposure and a slice thickness of 76 um to obtain a pretreatment outline of the root canals. All the scans were reoriented with respect to $\mathrm{x}, \mathrm{y}, \mathrm{z}$ axis. The canal transportation and volume of root canals was evaluated at 0.0, 1.0, 2.0, 3.0, 4.0, $5.0 \mathrm{~mm}$ intervals. The mean difference in Volume and Area was done using the One-ANOVA test with post-hoc bonferroni test for inter group comparisons.

Results: Twisted File Adaptive instruments did the least modifications in the Canal Area and Volume. P values of TFA was 0.001 , Wave One gold was $<0.001$.

Conclusion: Twisted File Adaptive maintained the original canal anatomy. Reciprocating instruments have a higher tendency for canals transportation as compared to Twisted File Adaptive.
\end{abstract}

Keywords: Root canal, Root canal preparation, Reciprocation.

\section{Introduction}

Root Canal Treatment is based on establishing an appropriate diagnosis and developing an appropriate treatment plan; applying knowledge of the tooth anatomy, shape and performing the debridement, disinfection and obturation of the entire root canal system. Canals which are poorly obturated are often incompletely shaped and cleaned. Adequate Cleaning and Shaping and establishing a coronal seal are the essential elements for successful root canal treatment.

New systems that use reciprocation motion are introduced to the market with the claim to shape root canals with only one file. As a result of technique simplification of technique according to their claims, clinicians can save time and cost for the endodontic treatment.

\section{Methodology}

The Aim of this Study was to evaluate the Shaping Characterstics of Adaptive File System and Reciprocating File System that is Twisted File Adaptive (Sybron Endo) and Wave One Gold (Dentsply Maillefer) file system. The Objective of the Study were to evaluate the Shaping Characterstics and to compare the Transportation and Residual Dentin Thickness in Mesial Curved Root Canals of Mandibular Molars using CBCT.

A Total of Sixty recently extracted intact and carious free Human Permanent Mandibular First Molars with mature apices were selected from the Department of Oral \& Maxillofacial Surgery, Seema Dental College \& Hospital for this study. Extraneous soft tissue, superficial Debris and Calculus were removed from the roots with Ultrasonic Scaler and teeth were then disinfected with 5.25 percentage of Concentrated Sodium Hypochlorite solution and were then Autoclaved at 137 Degree Celcius for Thirty minutes. Inclusion criteria demanded that the tooth had a curved mesial root with two separate canal and apices, with curvature angles ranging within $20-40^{\circ}$ (Schneider 1971). The teeth were examined under an operating surgical microscope to evaluate for the microcracks. All unacceptable teeth were discarded and selected teeth were stored in Deionized water under 4 degree Celcius until use.

RVG Radiographs were recorded in buccolingual and mesiodistal dimension before the instrumentation. In an attempt to standardize the length of root canal, teeth were decoronated at Cemento Enamel Junction using High Speed Alloy Grinder to get approx $14 \mathrm{~mm}$ length of Samples to confirm the presence of two distinct and separate root canals. In the mesiodistal dimension K-File no.10 were inserted into the buccal and lingual canals to assess the degree of root canal curvature according to Schneider's technique. Coronal access was achieved by using High Speed water cooling Airotor along with Endo-Access Bur to obtain a straight line access. Each tooth was sectioned through the furcation and mesial portion of the root and crown was used. Distal roots along with the part of crown were sectioned at the furcation level and were discarded.

Working Length was established in Sixty Specimens by deducting $1 \mathrm{~mm}$ from actual canal length which was determined by inserting No. $15 \mathrm{~K}, 21 \mathrm{~mm}$ Stainless Steel file into the canal until the tip of the file 
was just visible at the apical foramen. Working Length was confirmed with RVG Radiograph. In Both The File Systems that is Twisted File Adaptive NiTi Endodontic Adaptive System $(n=30)$ and Wave One Gold NiTi Endodontic System $(\mathrm{n}=30)$, Biomechanical Preparation was done according to Manufacturer's instructions.

Imaging of all the groups were done (90 micron resolution) with cone beam computed tomographic imaging software CS9300 equipment(Carestream Healthcare India(P) Ltd )set at $84 \mathrm{Kv}, 5 \mathrm{~mA}, 10.8$ second exposure and a slice thickness of 76 um to obtain a pretreatment outline of the root canals. The acquired images were three dimensionally reconstructed into cross sectional slices using standardized parameters for beam hardening and similar contrast limits. Extending from the furcation level to the apex of the root, resulting in the acquisition of 700-900 transverse cross section and dentin thickness from the level of the furcation to the apex of the root were recorded. All the scans were reoriented with respect to $\mathrm{x}, \mathrm{y}, \mathrm{z}$ axis. Study images were reconstructed from the volumetric data set in planes perpendicular to the selected tooth axis.

The teeth were then marked on the sides to differentiate the mesial and buccal sides, they were mounted on the acrylic block upto five in a block. Block was also marked to differentiate the front and back side of the block. The marked block with the teeth was placed into the CBCT for scanning.

Comarision parameters were:

a) The mean difference of superimposition at 1,2,3,4,5 mm in Root Canal Area from Pre to Post Treatment.

b) The mean difference of superimposition in Mesio Buccal and Mesio Lingual Canal Volume from Pre to Post Treatment.

c) The mean difference of superimposition in Mesial and Distal Root Angulation from Pre to Post Treatment.

Comparisons parameters were calculated by subtracting values obtain for treated canals with those from untreated canals through CBCT.

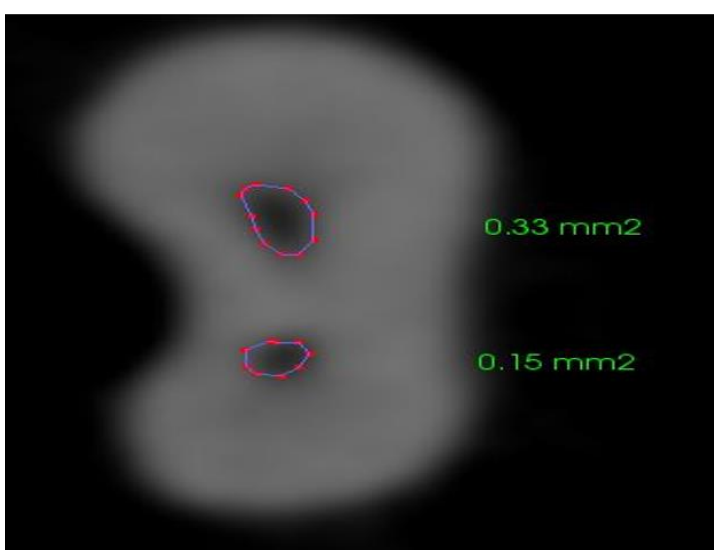

Fig. 1a: Pre-operative scan calculating area of the canal using CBCT

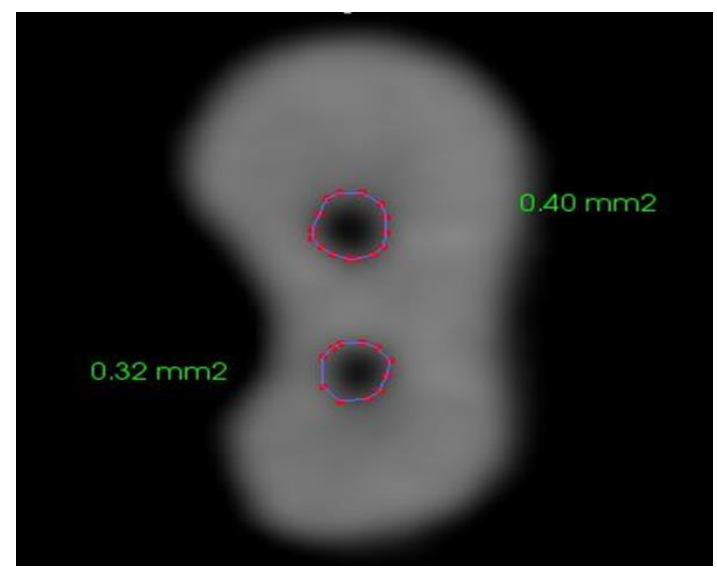

Fig. 1b: Post-operative scan calculating area of the canal using CBCT

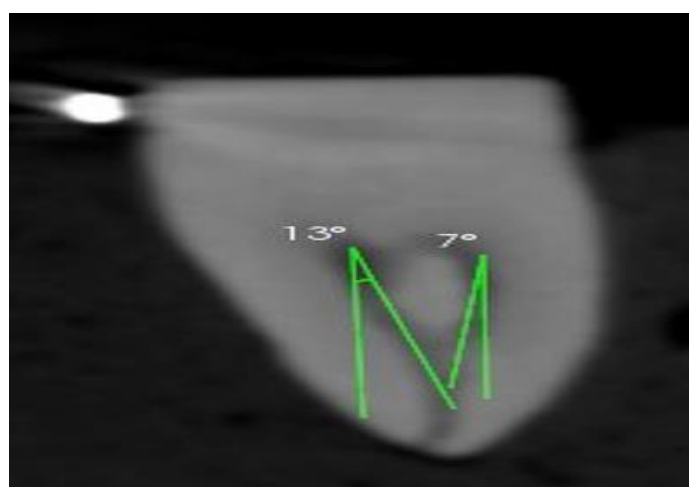

Fig. 2: Alculating canal angulation of mesiobuccal \& mesiolingual canal using CBCT

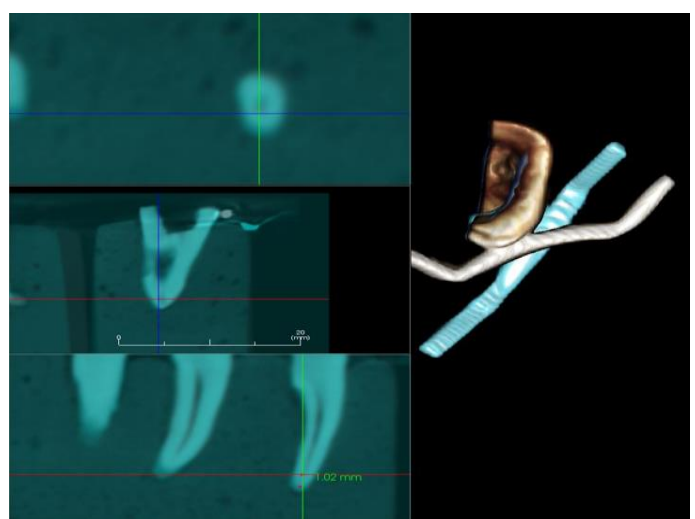

Fig. 3: Superimposition Images Using CBCT 


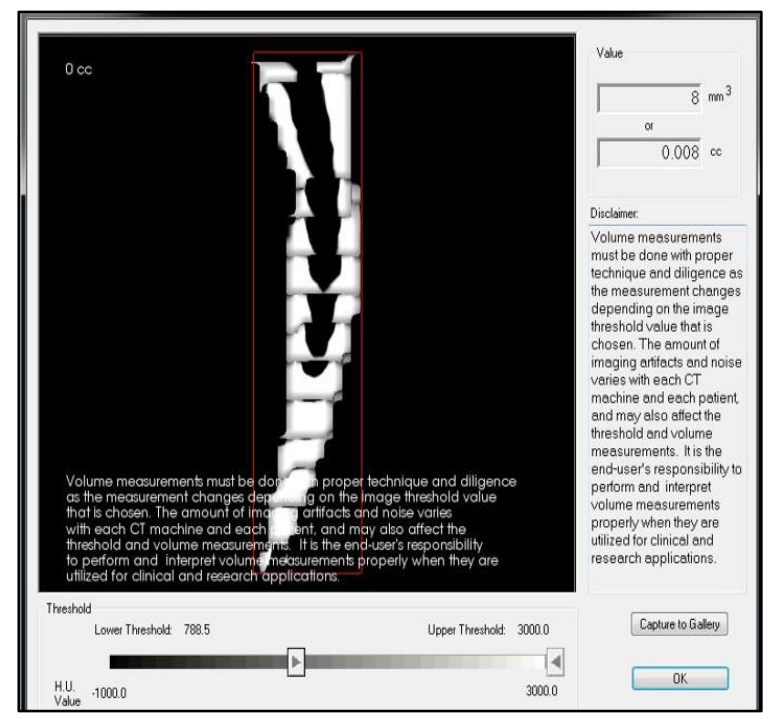

Fig. 4: Pre-operative scan calculating volume of the canal using CBCT

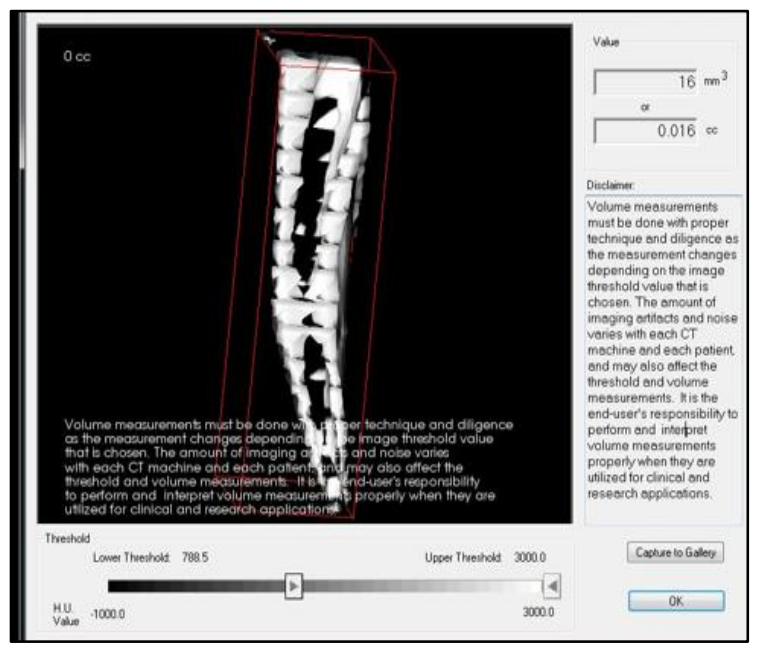

Fig. 5: Post-operative scan calculating volume of the canal using CBCT

\section{Statistical analysis}

For statistical analysis pos- hoc bonferroni test was used for the inter group comparisions and one-way anova test was used for intra group comparisions.

Descriptive statistics was performed by calculating mean, standard deviation, frequencies and percentages for the continuous variables. Categorical variables were summarized as frequencies and percentages.

The software used for the statistical analysis were SPSS (statistical package for social sciences) version 21.0 and epi-info version 3.0.

\section{Results}

Table 1: the mean difference of superimpositon at $1,2,3,4,5 \mathrm{~mm}$ in root canal area from pre to post treatment

\begin{tabular}{|c|c|c|c|c|}
\hline & \multicolumn{4}{|c|}{$\begin{array}{l}\text { Mean difference of Superimpositon at 1,2,3,4,5 mm in } \\
\text { Root Canal Area }\end{array}$} \\
\hline & Mean & Std. Deviation & F-value & P-value \\
\hline Group 1 & 11.36 & 0.124 & 5.764 & $0.001^{*}$ \\
\hline Group 2 & 0.06 & 0.032 & & \\
\hline
\end{tabular}

One-way ANOVA test *Significant difference

On Comparing Mean and Standard Deviation value in Table 2: Group 1(11.36 \pm 0.124$)$ Group 2(0.06 \pm 0.032$)$ and Group 3(0.058 \pm 0.032$)$ Group 1 showed maximum Area Recorded and Group 2 showed the least. 
Group 1 (twisted file adaptive)

Group 2 (wave one gold)

Table 2 (a): Difference in mesio-buccal volume from pre to post treatment

One-way ANOVA test

\begin{tabular}{|l|c|c|c|c|}
\hline & \multicolumn{4}{|l|}{$\begin{array}{l}\text { Difference in mesio-buccal volume from Pre } \\
\text { to Post treatment }\end{array}$} \\
\hline & Mean & Std. Deviation & F-value & p-value \\
\hline Group 1 & 15.80 & 2.59 & 14.993 & $0.001^{*}$ \\
\hline Group 2 & 9.20 & 2.28 & & \\
\hline
\end{tabular}

*Significantdifference

On Comparing Mean and Standard Deviation value

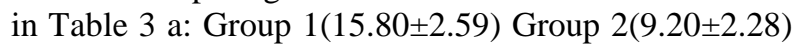

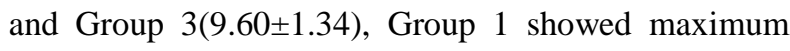
Volume Recorded and Group 2 showed the least.

Table 2: The Mean difference of canal volume in Superimposition of Mesio-Buccal and Mesio-Lingual Volume

One-way ANOVA test

\begin{tabular}{|c|c|c|c|c|}
\hline & \multicolumn{4}{|c|}{ The Mean difference of Canal Volume } \\
\hline & Mean & Std. Deviation & F-value & P-value \\
\hline Group 1 & 13.7 & 2.55 & 10.360 & $0.015^{*}$ \\
\hline Group 2 & 8.7 & 1.79 & & \\
\hline
\end{tabular}

* Significant difference

On Comparing Mean and Standard Deviation value in Table 4: Group 1(13.7 \pm 2.55$)$ Group 2(8.7 \pm 1.79$)$ and Group 3(8.7 \pm 1.49$)$ Group 1 showed maximum Volume Recorded and Group 2 showed the least.

Table 3: The mean difference of canal angulation in superimposition of mesial and distal root angulation

\begin{tabular}{|c|c|c|c|c|}
\hline & \multicolumn{4}{|c|}{ The Mean difference of Canal Angulation } \\
\hline & Mean & Std. Deviation & F-value & p-value \\
\hline Group 1 & 11.0 & 4.325 & 0.173 & 0.843 \\
\hline Group 2 & 9.7 & 5.035 & & \\
\hline
\end{tabular}

One-way ANOVA test

* Significant difference

On Comparing Mean and Standard Deviation value

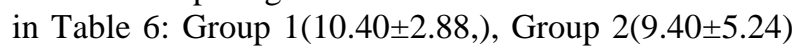
and Group 3(9.40 \pm 3.05$)$, Group 1 showed a significant difference followed and Group 2 showed the least.

Group 1 (Twisted File Adaptive)

Group 2 (Wave One Gold)

Table 4: Group 1 (twisted file adaptive) comparision of all parameters

\begin{tabular}{|c|c|c|}
\hline & Mean & $\begin{array}{c}\text { Std. } \\
\text { Deviation }\end{array}$ \\
\hline Area & 11.36 & 0.124 \\
\hline Volume & 13.7 & 2.55 \\
\hline Angulation & 11 & 4.325 \\
\hline
\end{tabular}

Table 5: Group 2(wave one gold)Comparision of all parameters

\begin{tabular}{|c|c|c|}
\hline & Mean & $\begin{array}{l}\text { Std. } \\
\text { Deviation }\end{array}$ \\
\hline AREA & 0.06 & 0.032 \\
\hline VOLUME & 8.7 & 1.79 \\
\hline ANGULATION & 9.7 & 5.035 \\
\hline
\end{tabular}

Group 1 (Twisted File Adaptive)

Group 2 (Wave One Gold)
The TF Adaptive technique is a three-file technique which is designed to treat the majority of cases that are encountered in clinical practice. Three-file systems in two sets are available -one for small calcified canals and one for more "standard" and larger canals. In both sequence of events, this allows requisite taper and increased apical preparation. The number of instruments within each concatenation can also vary and acclimatize to canal anatomy, with the last instrument of the sequence used only when a greater apical enlargement is required due to larger original canal dimensions or enhanced final irrigation techniques.

The medium/large canal sequence is a "true" crown- down technique, the small canal sequence employs a smaller more flexible instrument (04 taper 20 tip size) to pre- enlarge the canal and create a glide path, which decreases instrument strain for the next larger size file to sequence. The use of a final apical enlargement with a size 35 is not only suggested to allow the use of the Endo. Vac (SybronEndo) irrigation technique, but to enhance canal shaping by touching

\section{Discussion}


more canal walls. Reason being, why in the majority of cases, two instruments are much better than a single file technique, assuming that the second instrument is a flexible one. The superior flexibility and pliability allowed by the Twisted File technology enables TF Adaptive to follow these criteria and safely enlarge canals with a negligible risk of iatrogenic errors, such as tooth weakening and apical transportation.

Wave One Gold NiTi file system from DENTSPLY Maillefer is a single -use, single -file system to shape the root canal. Shaping the root canal to a continual tapering funnel shape not only attains the biological demands for adequate irrigation to free the root canal system of all bacteria, bacterial by-products and pulp tissue, but also bestow the perfect shape for 3D obturation with gutta-percha. The notably designed NiTi files work in a indistinguishable but reverse "balanced force" action using a pre-programmed motor to move the files in a back and forth "reciprocal motion". The files are using M-Wire technology, thereby improving strength and resistance to cyclic fatigue by up to nearly four times in comparison with other brands of rotary NiTi files. Wave One Gold file system has parallelogram cross section with two cutting edges. It features the off centered design of Protaper Next files and is manufactured with advanced gold heat treatment technique. This new manufacture technique improves the elasticity of file. ${ }^{53}$

There are three files in the Wave One Gold single file reciprocating system available in lengths of 21, 25 and $31 \mathrm{~mm}$. The Wave One Gold Small file is used in fine canals. The wave one gold primary file is used in the majority of canals. The Wave One Gold Large file is used in large canals. The instruments are designed to work with a reverse cutting action. All instruments have a improved convex triangular cross-section at the tip end. There is a possibility of cross-contamination kindered with the inability to completely clean and sterilise endodontic instruments and the possible presence of prion in human dental pulp tissue, all instruments used in root canals should be single use. Wave One Gold instruments are a new abstraction in this important standard of care, as they are single use. The plastic colour coding in the handle is deformed once sterilised, preventing the file from being placed back into the hand piece. The recommendation for single use has the added lead of minimizing instrument fatigue, which is an important consideration with Wave One Gold files, as one file does the work traditionally performed by three or more rotary NiTi files. The counterclockwise movement is greater than the clockwise movement. CCW movement advances the instrument while engaging and cutting the dentine. $\mathrm{CW}$ movement disengages the instrument from the dentine before it locks into the canal. Three reciprocating cycles complete one complete reverse rotation and the instrument moderately advances into the canal with little apical pressure required. ${ }^{52}$
The present Ex Vivo evaluated the shaping characteristics of the TF Adaptive and Wave One Gold File Systems using CBCT imaging, a nondestructive, reproducible, and well-established method for the 3D assessment of the root canal preparation.

The mean Difference in Area after superimposition showed highest values in Group $1(11.36 \pm 0.124)$ and least in Group $2(0.06 \pm 0.032)$

The mean area recorded pre and post for Group1 and Group 2 showed that Group 2 was not able to touch the walls of the canal while shaping. Better results were observed in Group 1 as the files touched the walls of the canals leading to proper cleaning and shaping of the canal. Overall, Group 1 systems resulted in significantly less unprepared root dentin canal walls and a higher increase in the surface area, perimeter, and minor diameter of the canals than the Group 2. These results can be explained by differences in the design and geometry of the instruments. The rationale behind measurement of changes in the cross sectional area was to enable comparisons at standardized cut planes. Results have shown that regardless of the reciprocating system used, the cross-sectional area increased at all levels. Nonetheless, there was no difference between any reciprocating systems at any cut plane.

The mean pre-instrumentation canal volumes were comparable, stipulating similar root canal sizes. The mesio-buccal and mesio-lingual canals were used as these canals are prone to iatrogenic errors because they are narrow and have accentuated curves that increase the level of instrumentation difficulty. Canal volume is a variable which is used to analyse the effects of canal instrumentation on dentine removal. Overinstrumentation of the root canal can result in excessive thinning of the root.

Mean difference in Canal Volume after superimposition images of Mesiobucal and Mesiolingual Canal showed highest values in Group 1 $(13.7 \pm 2.55)$ and least in Group $2(8.7 \pm 1.79)$.

Better results were observed in Group 1 as the files cleaned and shaped the root canal approaching the maximum walls of the canal. In this study, root canal instrumentation resulted in an increase in canal volume, which improves ingress of irrigants to the apical-third of the canal, but is also an indication that mechanical debridement might not be as successful apically as it is coronally.

According to the results of present study, it was determined that Group 1 file system which was used in the adaptive motion had statistically significant effect when compared with Group 2. The increase of TF NiTi file flexibility was reported to ensure the protection of original form of canal during root canal shaping. TF Adaptive niti files are manufactured using R-phase thus they are more flexible. In contrast to the result of present study Karatas (2016) et al examined the shaping abilities of TF NiTi files operated with different 
kinematics on the mesial canals of mandibular molar teeth by using Computed micro tomography

The mean difference of Canal Angulation in superimposition of Mesial and Distal root angulation showed higher values in Group $1(11 \pm 4.325)$ and lesser in Group 2 ( $9.7 \pm 5.035$ ).

Hence it was concluded that Group 1 was more superior in maintaining the original Canal anatomy as compared to Group 2. However large Sample size along with clinical trial is necessary to validate the results of present ex vivo study.

Within the limitations of this study long term use of this method should be followed up in clinical studies. However more ex vivo studies need to be conducted to correlate the result of this study.

\section{Conclusion}

The results of this Ex Vivo study regardless of the instrument used to perform shaping and cleaning, the cross-sectional area increased at all levels. In this study Twisted File Adaptive Files resulted in less transportation as compared to other group.

Within the parameters of this study it was concluded that Twisted File Adaptive Files did the least modifications in canal anatomy as compared to Wave One Gold File System.

Within the limitations of this study long term use of these method should be followed up in clinical studies. However more ex vivo studies need to be conducted to correlate the result of this study.

\section{References}

1. Frank AL, The reciprocating movement in endodontics. $J$ of Endod practice. 1967

2. Olav Molven, A comparison of the dentin-removing ability of five root canal instruments, National Center for Biotechnology Information. 1970.

3. Olav Molven, A comparison of the dentin-removing ability of five root canal instruments, $J$ of Endod practice. 1970.

4. Weine FS, Kelly RF, The effect of preparation procedures on original canal shape and on apical foramen shape, National Center for Biotechnology Information.1975.

5. Malentacca A, Lalli F, Evaluation of Effectiveness of Manual and Rotary Techniques in Root Canal System. NCBI. 2002.

6. Martin H, Endosonic Endodontics, National Center for Biotechnology Information. 1976.

7. Lehman JW, Effectiveness of Four Methods for Preparation of Root Canal System, National Center for Biotechnology Information. 1982.

8. James B. Roane, Clyde L. Sabala. The balanced force concept for instrumentation of curved canals. Int. Endodo J .1990.

9. Leubke NH, Brantley WA, Bending Fatigue Study of NiTi Gates Glidden, National Center for Biotechnology Information. 1990.

10. B.M. Briseno, E. Sonnabend, The influence of different root canal instruments on root canal preparation: an in vitro study. Wiley Online Library. 1991. (More References on request). 